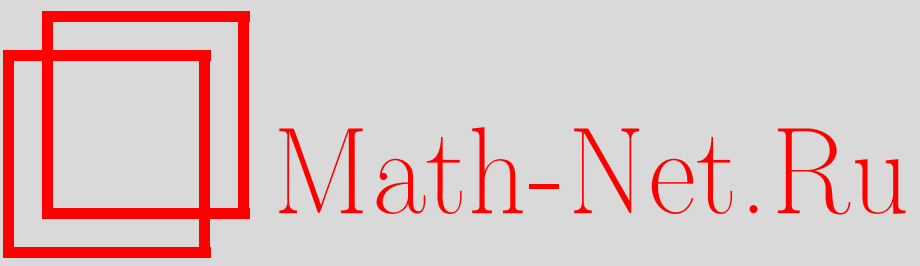

Т. А. Иоанниду, Нетривиальное рассеяние солитонов в $(2+1)$-мерном пространстве анти-де Ситтера, ТМФ, 2003, том 137, номер 1, 137-141

DOI: https://doi.org/10.4213/tmf251

Использование Общероссийского математического портала Math-Net.Ru подразумевает, что вы прочитали и согласны с пользовательским соглашением

http://www.mathnet.ru/rus/agreement

Параметры загрузки:

IP : 54.162 .85 .209

26 апреля 2023 г., $12: 40: 30$ 


\section{НЕТРИВИАЛЬНОЕ РАССЕЯНИЕ СОЛИТОНОВ В $(2+1)$-МЕРНОМ ПРОСТРАНСТВЕ АНТИ-ДЕ СИТТЕРА}

Интегрируемые решения уравнений Богомольного-Янга-Миллса-Хиггса в $(2+1)$ мерном пространстве анти-де Ситтера находятся с помощью аналитических методов. В частности, приводится явная конструкция для семейств солитонных решений, динамика которых исследуется в некоторых деталях.

Ключевые слова: солитоны, интегрируемость, пространство анти-де Ситтера.

\section{1. ВВЕДЕНИЕ}

В данной работе (более подробное изложение см. в [1]) рассматривается интегрируемая система [2], связанная с конструкцией гиперболических монополей. Исследуемая модель получается при замене положительно определенного пространства $\mathbb{H}^{3}$ в уравнениях гиперболических монополей на его лоренцев аналог, т.е. на пространство анти-де Ситтера. Версия Богомольного уравнений Янга-Миллса-Хиггса для полей Янга-Миллса-Хиггса на трехмерном римановом многообразии $\mathcal{M}$ с калибровочной группой $S U(2)$ имеет вид

$$
D_{i} \Phi=\frac{1}{2 \sqrt{|g|}} g_{i j} \epsilon^{j k l} F_{k l},
$$

где $A_{k}, k=0,1,2,-$ калибровочный потенциал, принимающий значения в алгебре $s u(2)$ с тензором поля $F_{i j}=\partial_{i} A_{j}-\partial_{j} A_{i}+\left[A_{i}, A_{j}\right], \Phi=\Phi\left(x^{\mu}\right)$-(скалярное) поле Хиггса, также принимаюшее значения в алгебре $s u(2)$, а $x^{\mu}=\left(x^{0}, x^{1}, x^{2}\right)$ - локальные координаты на многообразии $\mathcal{M}$. Ковариантная производная $D_{i}=\partial_{i}+A_{i}$ действует на поле $\Phi$ по правилу $D_{i} \Phi=\partial_{i} \Phi+\left[A_{i}, \Phi\right]$. Уравнение (1) интегрируемо в том смысле, что в случае постоянной кривизны существует пара Лакса. В частности, решения уравнения (1) отвечают евклидовым или гиперболическим монополям Богомольного-Прасада-Зоммерфельда в зависимости от того, каким пространством локально является многообразие $(\mathcal{M}, g)$ : евклидовым $\mathbb{R}^{3}$ или гиперболическим $\mathbb{H}^{3}$.

По определению $(2+1)$-мерное пространство анти-де Ситтера представляет собой универсальное накрытие гиперболоида $\mathcal{H}$, задаваемого уравнением

$$
U^{2}+V^{2}-X^{2}-Y^{2}=1
$$

* Institute of Mathematics, University of Kent at Canterbury, Canterbury CT2 7NF, UK. E-mail: T.Ioannidou@ukc.ac.uk 
где метрика имеет вид

$$
d s^{2}=-d U^{2}-d V^{2}+d X^{2}+d Y^{2}
$$

Вводя параметризацию гиперболоида $\mathcal{H}$

$$
\begin{aligned}
& U=\sec \rho \cos \theta, \\
& V=\sec \rho \sin \theta, \\
& X=\operatorname{tg} \rho \cos \phi, \\
& Y=\operatorname{tg} \rho \sin \phi,
\end{aligned}
$$

получим, что при $\rho \in[0, \pi / 2)$ соответствующая метрика примет вид

$$
d s^{2}=\sec ^{2} \rho\left(-d \theta^{2}+d \rho^{2}+\sin ^{2} \rho d \phi^{2}\right) .
$$

Пространство-время содержит замкнутые времениподобные кривые ввиду периодичности переменной $\theta$. На самом деле пространство анти-де Ситтера (как многообразие) представляет собой произведение открытого пространственного диска на переменную $\theta$ и имеет постоянную кривизну, равную минус шести, где $(\rho, \phi)$ соответствуют полярным координатам, а переменная $\theta \in \mathbb{R}$ играет роль времени. Исчезающая пространственная бесконечность $\mathcal{I}$ представляет собой времениподобный цилиндр $\rho=\pi / 2$, и эта поверхность никогда не достигается никакой времениподобной геодезической.

Если задать координаты Пуанкаре $(r, x, t)$ при $r>0$ в виде

$$
r=\frac{1}{U+X}, \quad x=\frac{Y}{U+X}, \quad t=\frac{-V}{U+X},
$$

то выражение для метрики упростится:

$$
d s^{2}=r^{-2}\left(-d t^{2}+d r^{2}+d x^{2}\right) .
$$

Заметим, что координаты Пуанкаре покрывают лишь малую область пространства анти-де Ситтера, а именно ту его часть, которая отвечает половине гиперболоида $\mathcal{H}$ при $U+X>0$. Поверхность $r=0$ представляет собой часть пространственной бесконечности $\mathcal{I}$.

Рассмотрим набор линейных уравнений

$$
\begin{aligned}
{\left[r D_{r}-2(\lambda-u) D_{u}-\Phi\right] \Psi } & =0 \\
{\left[2 D_{v}+\frac{\lambda-u}{r} D_{r}-\frac{\lambda-u}{r^{2}} \Phi\right] \Psi } & =0
\end{aligned}
$$

где $\lambda \in \mathbb{C}$ и $r, u=x+t, v=x-t$ суть координаты Пуанкаре. Калибровочные поля $\left(\Phi, A_{r}, A_{u}, A_{v}\right)$ задаются $(2 \times 2)$-матрицами с нулевым следом, зависяшими только от переменных $r, u, v$, а $\Psi(\lambda, r, u, t)$ есть унимодулярная $(2 \times 2)$-матричнозначная функция, удовлетворяющая условию вешественности $\Psi(\lambda) \Psi(\bar{\lambda})^{\dagger}=I$ (где крест обозначает транспонирование с одновременным комплексным сопряжением). Система уравнений (4) оказывается переопределенной, и для сушествования ее решения $\Psi$ должны быть выполнены следуюшие условия интегрируемости:

$$
\begin{aligned}
D_{u} \Phi & =r F_{u r}, \\
D_{v} \Phi & =-r F_{v r}, \\
D_{r} \Phi & =-2 r F_{u v} .
\end{aligned}
$$


Из пары Лакса (4) можно получить калибровочные поля и поле Хиггса в терминах функции $\Psi$. Заметим, что в пределе $\lambda \rightarrow \infty$ функция $\Psi$ стремится к единичной матрице, что приводит к условиям

$$
A_{u}=0, \quad A_{r}=\frac{1}{r} \Phi .
$$

С другой стороны, при $\lambda=0$ остаюшиеся калибровочные поля, задаваемые выражениями (6), имеют вид

$$
\begin{aligned}
\Phi & =-\frac{r}{2} J_{r} J^{-1}-u J_{u} J^{-1}, \\
A_{v} & =\frac{u}{2 r} J_{r} J^{-1}-J_{v} J^{-1},
\end{aligned}
$$

где $J(r, u, v) \doteq \Psi(\lambda=0, r, u, v)$. Заметим, что в этом случае первое из уравнений системы (5) выполняется автоматически (ввиду специального выбора калибровки). Как было показано Уордом [2], голоморфные векторные расслоения $V$ над многообразием $Q$ задают с помошью стандартных преобразований Пенроуза мультисолитонные решения системы (5) в пространстве анти-де Ситтера. При этом так же, как и в случае плоского пространства-времени [3], получается пятипараметрическое семейство солитонных решений.

\section{2. РАССЕЯНИЕ СОЛИТОНОВ}

В рамках стандартного метода задачи Римана с нулями, применяемого при построении мультисолитонных решений, будем предполагать [3], что функция $\Psi$ имеет простой вид относительно переменной $\lambda$ :

$$
\Psi=I+\sum_{k=1}^{n} \frac{M_{k}}{\lambda-\mu_{k}},
$$

где $M_{k}$ суть $(2 \times 2)$-матрищы, не зависяшие от $\lambda$, а $n$ - число солитонов. Компоненты матрицы $M_{k}$ задаются с помощью рациональной функции $f_{k}\left(\omega_{k}\right)=a_{k} \omega_{k}+c_{k}$ от комплексного аргумента $\omega_{k}=v-r^{2}\left(\mu_{k}-u\right)^{-1}$. Здесь $a_{k}, c_{k}$ и $\mu_{k}-$ комплексные константы, задающие размер, положение и скорость $k$-го солитона.

Матрица $M_{k}$ имеет вид

$$
M_{k}=\sum_{l=1}^{n}\left(\Gamma^{-1}\right)^{k l} \bar{m}_{a}^{l} m_{b}^{k},
$$

где $\Gamma^{-1}$ есть матрица, обратная матрице

$$
\Gamma^{k l}=\sum_{a=1}^{2}\left(\bar{\mu}_{k}-\mu_{l}\right)^{-1} \bar{m}_{a}^{k} m_{a}^{l},
$$

а $m_{a}^{k}$ - голоморфные функции переменных $\omega_{k}$ вида $m_{a}^{k}=\left(m_{1}^{k}, m_{2}^{k}\right)=\left(1, f_{k}\right)$. При этом поля Янга-Миллса-Хиггса $\Phi, A_{r}, A_{v}, A_{u}$ могут быть получены непосредственно из представлений (6) и (7), и они при этом автоматически удовлетворяют системе уравнений (5). Соответствуюшие солитоны оказываются пространственно локализованными, 
поскольку $\Phi \rightarrow 0$ в пространственной бесконечности (т.е. при $r=0$ ). В этом случае рассеяние солитонов тривиально, т.е. они проходят один сквозь другой, испытывая нулевые фазовые сдвиги и не изменяя ни своей скорости, ни своей формы.

Метод задачи Римана с нулями предполагает, что параметры $\mu_{k}$ не равны друг другу, а также что $\bar{\mu}_{k} \neq \mu_{l}$ при всех $k, l$. Тем не менее в примерах, обобщающих эти построения, либо допускаются полюсы старшего порядка по переменным $\mu_{k}$, либо возможны ситуации, когда $\bar{\mu}_{k}=\mu_{l}$. При применении этой процедуры к плоскому пространству-времени соответствуюшие солитоны рассеиваются нетривиально. В частности, при лобовых соударениях $N$ неразличимых солитонов угол рассеяния возникающих солитонов по отношению к сталкивающимся равен $\pi / N[4],[5]$.

Рассмотрим сначала пример, в котором функция $\Psi$ имеет полюс второго порядка по переменной $\lambda$ и не имеет других полюсов. Тогда функция $\Psi$ может быть записана в виде

$$
\Psi=I+\sum_{k=1}^{2} \frac{R_{k}}{(\lambda-\mu)^{k}}
$$

где $R_{k}$ суть $(2 \times 2)$-матирцы, не зависяшие от $\lambda$. При этом, как и в случае плоского пространства-времени [4], функция $\Psi$ задает решение системы (4) тогда и только тогда, когда она может быть факторизована в виде

$$
\Psi(\lambda)=\left(1-\frac{\bar{\mu}-\mu}{(\lambda-\mu)} \frac{q^{\dagger} \otimes q}{|q|^{2}}\right)\left(1-\frac{\bar{\mu}-\mu}{(\lambda-\mu)} \frac{p^{\dagger} \otimes p}{|p|^{2}}\right)
$$

для некоторых двух векторов $q$ и $p$. Один из способов найти вид этих векторов состоит в том, чтобы рассмотреть формулу (8) при $n=2$ и положить $\mu_{1}=\mu+\epsilon, \mu_{2}=\mu-\epsilon$, $f_{1}\left(\omega_{1}\right)=f\left(\omega_{1}\right)+\epsilon h\left(\omega_{1}\right)$ и $f_{2}\left(\omega_{2}\right)=f\left(\omega_{2}\right)-\epsilon h\left(\omega_{2}\right)$, предполагая, что функции $f$ и $h$ суть рациональные функции от одной переменной. В пределе $\epsilon \rightarrow 0$ векторы $q$ и $p$ имеют вид

$$
\begin{aligned}
& q=\left(1+|f|^{2}\right)(1, f)+(\bar{\mu}-\mu)\left(\frac{r^{2} f^{\prime}}{(\mu-u)^{2}}+h\right)(\bar{f},-1), \\
& p=(1, f) .
\end{aligned}
$$

В этом случае следует наложить условие $f_{2}\left(\omega_{2}\right)-f_{1}\left(\omega_{1}\right) \rightarrow 0$ при $\epsilon \rightarrow 0$, чтобы обеспечить гладкость получаюшегося решения $\Psi$ при всех $r, u, v$. В простейшем случае, когда $\mu=i, f(\omega)=\omega$ и $h(\omega)=0$, величина $-\operatorname{tr} \Phi^{2}$ имеет простой вид

$$
-\operatorname{tr} \Phi^{2}=32 r^{2} \frac{\left[\left(r^{2}+x^{2}-t^{2}+1\right)^{2}+4 t^{2}\right]\left[\left(r^{2}+x^{2}-t^{2}-1\right)^{2}+4 x^{2}\right]}{\left\{\left[\left(r^{2}+x^{2}-t^{2}\right)^{2}+1+2 t^{2}+2 x^{2}\right]^{2}+4 r^{4}\right\}^{2}},
$$

который допускает обрашение направления времени. Зависящее от времени решение представляет собой при этом движущуюся солитонную конфигурацию, перемешающуюся в направлении пространственной бесконечности $(r=0)$ при отрищательных $t$, достигающую ее при $t=0$, а затем отражающуюся назадпри положительных $t$. В течение этого процесса солитонная конфигурация деформируется.

В качестве второго примера рассмотрим обширное семейство решений, отвечаюших случаю $\bar{\mu}_{k}=\mu_{l}$. Один из методов рассмотрения данной ситуации состоит в том, чтобы 
взять решение (8) при $n=2$, положить $\mu_{1}=\mu+\epsilon$ и $\mu_{2}=\bar{\mu}-\epsilon$ и затем сделать предельный переход $\epsilon \rightarrow 0$. Для того чтобы обеспечить гладкость получаемого решения $\Psi$, необходимо выбрать $f_{1}\left(\omega_{1}\right)=f\left(\omega_{1}\right)$ и $f_{2}\left(\omega_{2}\right)=-1 / f\left(\omega_{2}\right)-\epsilon h\left(\omega_{2}\right)$, где $f$ и $h$ суть рациональные функции одной переменной. Вычисляя теперь предел, можно получить решение $\Psi$ в виде

$$
\Psi=I+\frac{n^{1} \otimes m^{1}}{\lambda-\mu}+\frac{n^{2} \otimes m^{2}}{\lambda-\bar{\mu}},
$$

где $n^{k}$ и $m^{k}, k=1,2,-$ комплекснозначные двухвекторные функции вида

$$
\begin{gathered}
m^{1}=(1, f), \quad m^{2}=(-\bar{f}, 1), \\
\left(\begin{array}{l}
n^{1} \\
n^{2}
\end{array}\right)=\frac{2(\mu-\bar{\mu})}{4\left(1+|f|^{2}\right)^{2}-(\mu-\bar{\mu})^{2}|w|^{2}}\left(\begin{array}{cc}
2\left(1+|f|^{2}\right) & -(\mu-\bar{\mu}) \bar{w} \\
(\mu-\bar{\mu}) w & -2\left(1+|f|^{2}\right)
\end{array}\right)\left(\begin{array}{l}
m^{1 \dagger} \\
m^{2 \dagger}
\end{array}\right)
\end{gathered}
$$

с параметром

$$
w \equiv \frac{2 r^{2}}{(\mu-u)^{2}} f^{\prime}+\bar{h} f^{2} .
$$

Таким образом, получено решение, зависящее от одного свободного параметра $\mu$ и от двух произвольных рациональных функций $f=f(\omega)$ и $h=h(\bar{\omega})$. При выборе $\mu=i$, $f=\omega$ и $h=\bar{\omega}$ получается конфигурация, состоящая из двух солитонов с нетривиальным поведением при рассеянии. Величина $-\operatorname{tr} \Phi^{2}$ снова имеет пиковые особенности в двух точках при отрицательных $t$, которые остаются различными и при $t=0$, а затем при (положительных) временах $t$ возникают два сдвинутых (по сравнению с исходными в момент времени $t=-3$ ) солитона. Форма этих солитонов также изменяется в процессе эволюции во времени.

\section{3. ЗАКЛЮЧЕНИЕ}

Таким образом, мы рассмотрели построение зависяших от времени решений, связанных с конструкцией гиперболических монополей. В частности, были построены семейства решения для версии Богомольного уравнений Янга-Миллса-Хиггса в $(2+1)$ мерном пространстве анти-де Ситтера и исследованы некоторые вопросы динамики построенных решений.

Было бы интересно понять роль полюсов старших порядков в алгебро-геометрическом подходе типа теории твисторов, а также исследовать построение соответствующих решений и их динамику в пространстве де Ситтера. Наконец, было бы интересно расширить данное построение на случай калибровочных теорий в старших размерностях и исследовать процесс рассеяния соответствуюших классических решений, равно как рассмотреть и изучить их некоммутативные обобщения (см., например, [6]).

\section{Список литературы}

[1] T. Ioannidou. Nonlinearity. 2002. V. 15. P. 1489.

[2] R. S. Ward. Asian J. Math. 1999. V. 3. P. 325.

[3] R. S. Ward. J. Math. Phys. 1988. V. 29. P. 386.

[4] R. S. Ward. Phys. Lett. A. 1995. V. 208. P. 203.

[5] T. Ioannidou. J. Math. Phys. 1996. V. 37. P. 3422.

[6] O. Lechtenfeld, A. D. Popov. Phys. Lett. B. 2001. V. 523. P. 178; hep-th/0108118. 